Vera Eichenauer und Jan-Egbert Sturm*

\title{
Die wirtschaftspolitischen Maßnahmen der Schweiz zu Beginn der Covid-19-Pandemie
}

https://doi.org/10.1515/pwp-2020-0027

Zusammenfassung: Die Schweizer Volkswirtschaft bewegte sich zu Beginn der Covid-19-Pandemie aus einem konjunkturellen Tief in eine sanfte Aufschwungsphase mit im internationalen Vergleich niedriger Arbeitslosigkeit und gesunden öffentlichen Finanzen. Aufgrund ihrer geographischen Lage waren insbesondere die italienisch- und französischsprachigen Regionen der Schweiz frühzeitig und stark von Ansteckungen mit SARS-CoV-2 betroffen. Während der Sperrperiode (sanfter Lockdown) hatte die Strukturerhaltung höchste Priorität. Arbeitsplätze wurden durch Kurzarbeit erhalten, die Liquidität der Unternehmen wurde durch den einfachen und raschen Zugang zu günstigen und staatlich garantierten Krediten gewährleistet. Im Hinblick auf die Prävention der Überschuldung der Wirtschaft und die für Innovations- und Investitionsentscheidungen relevante Erwartungsbildung wurde allerdings wenig unternommen. Unternehmen erhielten keine nennenswerte Unterstützung für ihre Kapitalkosten. In der Kommunikation der Regierung fehlte es zudem oft an vorwärtsorientierenden Aussagen.

JEL-Klassifikation: E60, E65, I18

Schlüsselwörter: Corona, Schweiz, Wirtschaftspolitik

Abstract: At the beginning of the Covid-19 pandemic, the Swiss economy was moving from an economic low into a gentle upswing phase with low unemployment and healthy public finances by international standards. Due to its geographic location, Switzerland and in particular its Italianand French-speaking regions were affected early and severely by SARS-CoV-2 infections. During the (gentle) lockdown period, the preservation of structures had top priority. Jobs were maintained by short-time work and the liquidity of

Hinweis: Dieser Beitrag spiegelt den Informationsstand vom 1. Mai 2020.

Vera Eichenauer, ETH Zürich, KOF Konjunkturforschungsstelle, Leonhardstrasse 21, CH-8092 Zürich,

E-Mail: eichenauer@kof.ethz.ch

*Kontaktperson: Jan-Egbert Sturm, ETH Zürich, KOF

Konjunkturforschungsstelle, Leonhardstrasse 21, CH-8092 Zürich,

E-Mail: sturm@kof.ethz.ch enterprises was upheld by easy and quick access to cheap and state-guaranteed credit. In contrast, little was done to prevent over-indebtedness of the economy and for firms to form expectations relevant for innovation and investment decisions. Companies received no significant support for bearing their cost of capital. The communication of government often lacked forward guidance.

\section{Die Covid-19-Pandemie in der Schweiz und die staatlichen Eindämmungsmaßnahmen}

Am 25. Februar 2020 bestätigte die Schweiz den ersten Fall von Covid-19; er betraf einen 70-jährigen Mann im italienischsprachigen, an Italien grenzenden Kanton Tessin. Der Mann hatte zuvor Mailand besucht und wurde positiv auf SARS-CoV-2 getestet. Aufgrund ihrer Nähe zu Norditalien war die Schweiz damit nach Italien eines der ersten Länder in Europa, das von der Covid-19-Pandemie erreicht wurde. Die Geographie erklärt wahrscheinlich auch, weshalb insbesondere das Tessin so stark betroffen ist und die Pro-Kopf-Fallzahlen der Schweiz innerhalb Europas zu den unerfreulichen „Spitzenwerten“ zählen.

Als Reaktion auf die rasch ansteigenden nationalen Fallzahlen ergriff der Bundesrat, die Schweizer Konsensregierung mit sieben Mitgliedern aus vier Parteien, ab Ende Februar eine Reihe von national einheitlichen Gesundheitsmaßnahmen und wirtschaftspolitischen Unterstützungsprogrammen. Kantonale Ausnahmen wurden nur im Einzelfall und in Rücksprache mit dem Bund, der Regierung auf Bundesebene, toleriert. Gestützt auf das Epidemien-Gesetz (EpG, AS 2015 1435), kann der Bundesrat unter Einbezug der Kantone die Ziele und Strategien der Erkennung, Überwachung, Verhütung und Bekämpfung übertragbarer Krankheiten festlegen. Die Schweizer Bevölkerung hatte das revidierte Gesetz 2013 in einem fakultativen Referendum mit einem Ja-Anteil von 60 Prozent angenommen, was den Rückhalt der nationalen Maßnahmen in der Bevölkerung und der Politik noch verstärkt haben dürfte.

Die von der Schweiz ergriffenen Einschränkungen des Wirtschaftslebens sind im Vergleich mit den meisten In- 


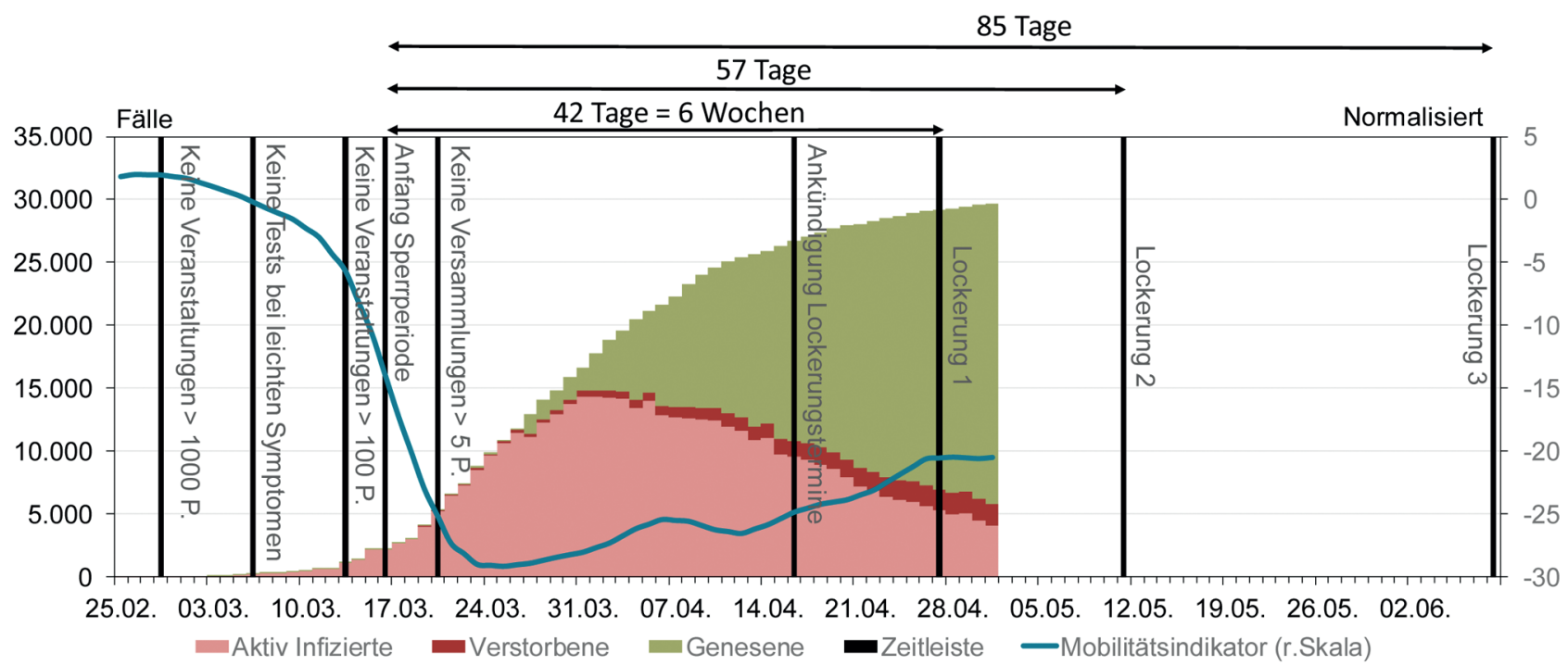

Abbildung 1: Die Entwicklung der Covid-19-Pandemie in der Schweiz und ein Mobilitätsindikator für die Schweiz Anmerkung: Der Mobilitätsindikator zeigt die grundlegende Tendenz von ausgewählten hochfrequenten Zeitreihen, die das Mobilitätsverhalten der Schweizer Bevölkerung widerspiegeln. Dazu gehören unter anderem das Verkehrsaufkommen im Individualverkehr, die Benutzung des öffentlichen Verkehrs, Bargeldbezüge, Debitkarten-Einsätze, Flugbewegungen und die Nutzung von Online-Plattformen. Der Mobilitätsindikator hat während der Periode vom 7. Januar bis 24. Februar 2020 einen Mittelwert von 0 und eine Standardabweichung von 1. Quelle: Johns Hopkins University, KOF

dustriestaaten mild. Während des Schweizer Lockdowns konnten die Industriebetriebe und das Baugewerbe (außer in wenigen Kantonen) unter Einhaltung der Hygienevorschriften weiterarbeiten. Der Alltag vieler Arbeitnehmer war durch die Arbeit und den Schulunterricht von zu Hause, die Beschränkung von Versammlungen auf maximal fünf Personen mit zwei Metern Distanz und der Schließung von Läden und Restaurationsbetrieben zwar sehr eingeschränkt, aber weniger stark als in den Nachbarländern. Diese verhältnismäßig geringen Einschränkungen, insbesondere für Firmen mit hoher Wertschöpfung, erklären, wieso in den Schätzungen der KOF Konjunkturforschungsstelle vom 7. und 24. April 2020 für die meisten Szenarien des Pandemieverlaufs in der Schweiz deutlich mehr als die Hälfte aller kurzfristigen Wertschöpfungsverluste mit internationalen Nachfragerückgängen und Lieferengpässen zusammenhängen. ${ }^{1}$

Der Bundesrat ergriff zunächst langsam und dann ab Mitte März 2020 in rasch aufeinanderfolgenden Teilschritten immer stärkere gesundheitspolitische Maßnahmen mit entsprechenden Konsequenzen für das Wirtschaftsleben. Gleichzeitig appellierte die Schweizer Regierung an die Eigenverantwortung und Rücksichtnahme der Bevölkerung und empfahl das Arbeiten von zu Hause. Zeitlich hinkte die Schweiz trotz hoher absoluter und Pro-KopfFallzahlen jeweils leicht hinter den Ankündigungen von

1 Rathke et al. 2020a, b.
Maßnahmen in den Nachbarländern her. Abbildung 1 zeigt die Entwicklung der Fallzahlen und der wichtigsten Maßnahmen. Zunächst wurden am 28. Februar Veranstaltungen mit mehr als 1000 Personen verboten. Anfangs wurde telefonisch versucht herauszufinden, mit wem infizierte Personen Kontakt gehabt hatten (Contact tracing). Hierzu riefen speziell ausgebildete Personen die Betroffenen an. Sobald die Zahl der Infektionen zunahm, funktionierte dieses System nicht mehr. Am 6. März wechselte der Bundesrat die „Strategie“ und gab die Rückverfolgung der Kontakte mit Infizierten auf. Zudem wurde klar, dass die Testkapazitäten nicht ausreichten, um die stark gestiegene Nachfrage zu befriedigen. Personen mit milden Symptomen wurden ab diesem Zeitpunkt nicht mehr getestet.

Nach diesen anfänglich zurückhaltenden Maßnahmen beschloss der Bundesrat am 13. März, den Präsenzunterricht in allen Bildungseinrichtungen einzustellen, und untersagte alle (öffentlichen und privaten) Veranstaltungen mit mehr als 100 Personen. Zudem durften sich in Speiseund Trinklokalen nur noch maximal 50 Personen aufhalten. Die Arbeitgeber wurden aufgefordert, besonders gefährdeten Arbeitnehmern die Möglichkeit zu geben, ihren Arbeitsverpflichtungen von zu Hause aus nachzukommen. Die Grenzen wurden teilweise geschlossen und Grenzkontrollen für Personen aus Risikogebieten eingeführt. ${ }^{2}$

2 Manche Kantone haben früher oder gleichzeitig drastischere Massnahmen ergriffen. Im Kanton Waadt wurden zum Beispiel gleichzeitig 
Gleichzeitig kündigte der Bundesrat (2020a) das erste wirtschaftliche Maßnahmenpaket im Umfang von 10 Milliarden Franken an, das sich mit 8 Milliarden Franken schwergewichtig auf die Kurzarbeit konzentrierte, aber auch ein Bürgschaftsprogramm über 580 Millionen enthielt.

Nur drei Tage später, am 16. März, wurde die außerordentliche Lage verkündet sowie Grenzkontrollen zu Frankreich, Italien und Österreich eingeführt. Restaurants, Bars sowie Unterhaltungs- und Freizeitbetriebe mussten schließen. Geöffnet bleiben durften Lebensmittelgeschäfte, Apotheken und die Post. Das Angebot des öffentlichen Verkehrs wurde reduziert. Die Baubranche und die Industrie wurden in der bundesrätlichen Notverordnung nicht erwähnt. Stark betroffene Kantone wie Genf und Waadt reagierten darauf und gestatteten nur Unternehmen, welche die Vorschriften über Hygiene und Sicherheitsabstand einhalten konnten, die Weiterführung des Betriebs. Am 18. März wurden Einreisebeschränkungen für NichtSchengen-Staaten und stark vom Corona-Virus betroffenen Schengen-Staaten verkündet. Am 20. März verschärfte die Regierung das Versammlungsverbot auf maximal fünf Personen und stockte die wirtschaftlichen Maßnahmen mit einer starken Ausweitung des Bürgschaftsprogramms auf insgesamt 42 Milliarden Franken auf. Die Einreisebeschränkungen wurden am 25. März auf alle SchengenStaaten ausgedehnt. Abbildung 1 zeigt, dass die Mobilität der Schweizer Bevölkerung bereits vor den einschneidenden Sperrmaßnahmen stark zurückgegangen war.

Am 3. April wurde das Bürgschaftsprogramm der Regierung von 20 auf 40 Milliarden Franken weiter erhöht. Um die wirtschaftlichen Konsequenzen der Verbote und Maßnahmen abzufedern, hat der Bundesrat somit bis zum 14. April 2020 Maßnahmenpakete im kumulativen Wert von 62 Milliarden Franken aufgelegt (das entspricht 9 Prozent des Schweizer BIP von 2019). Mehrmals sagten die Mitglieder des Bundesrats in den Pressekonferenzen von Ende März und Anfang April, dass eine substanzielle Lockerung der Lockdown-Maßnahmen bis Mitte April nicht realistisch sei. Am 8. April verkündete die Regierung schließlich eine Verlängerung der Maßnahmen bis zum 26. April, wobei sie für die Zeit danach einen schrittweisen Ausstieg in Aussicht stellte.

Am 16. April 2020 kündigte der Bundesrat an, dass er die Beschränkungen in drei Schritten unter Hygieneauflagen lockern werde. Über die Lockerung der Verbote und Beschränkungen für Gastronomie, Tourismus sowie Sportund Kulturangebote wurden zu diesem Zeitpunkt keine

alle öffentlichen und privaten Versammlungen mit mehr als 50 Personen verboten und Bildungseinrichtungen geschlossen.
Angaben gemacht. Die erste Stufe der Lockerung hat am 27. April begonnen, und zwar für diejenigen Berufe, in denen Menschen in engem Kontakt mit anderen, aber nicht in großer Zahl, arbeiten. Ärzte durften wieder nichtdringliche Behandlungen durchführen; Baumärkte, Blumenläden, Gartenzentren, Gärtnereien, Friseurläden sowie Kosmetik- und Massagestudios durften unter Einhaltung der Gesundheitsvorschriften wieder öffnen. Die Lockerungen werden von Schutzkonzepten begleitet, welche die Branchenverbände zu entwickeln und zur Prüfung vorzulegen hatten und haben. Ab dem 11. Mai sollen in einem zweiten Schritt die obligatorischen Schulen sowie alle Läden wieder öffnen. In einem dritten Schritt dürfen ab dem 8. Juni Mittel-, Berufs- und Hochschulen, sowie Museen, Zoos und Bibliotheken wieder öffnen. Bedingung ist immer, dass die Infektionslage es erlaubt.

Am 29. April gab der Bundesrat Lockerungen für weitere Branchen, beispielsweise Restaurants, ab dem 11. Mai bekannt. Zuvor war der Druck der Wirtschaftsverbände und der besonders wirtschaftsfreundlichen Parteien gestiegen. Erstens sollten weitere Sperrmaßnahmen so rasch wie möglich aufgehoben werden und zweitens sei Klarheit für Eventveranstalter zu schaffen. Parallel zu diesen Öffnungsschritten werden auch die Einreisebeschränkungen gelockert. Spätestens ab dem 11. Mai sollen zudem alle Kantone die flächendeckende Rückverfolgung von Neuinfektionen wiederaufnehmen, die in den meisten Kantonen ausgesetzt worden war.

\section{Wirtschaftliche Ausgangslage}

Bevor wir näher auf die wirtschaftlichen Folgen der Krise und die fiskalischen Maßnahmen eingehen, wollen wir zunächst kurz die wirtschaftliche Situation beschreiben, die vor dem Ausbruch der Covid-19-Pandemie in der Schweiz herrschte.

Die Schweiz war als kleine offene Volkswirtschaft in der Mitte Europas besonders im Finanzsektor und in der verarbeitenden Industrie von der globalen Finanzkrise 2008/09 und der anschließenden europäischen Schuldenkrise erschüttert worden. Dabei spielte nicht nur das negative internationale Umfeld eine wichtige Rolle, sondern auch die starke Aufwertung der Fluchtwährung Schweizer Franken. Dieser wertete real effektiv zwischen 2006 und 2011 um mehr als 20 Prozent auf. Erst die Einführung eines Mindestwechselkurses gegenüber dem Euro am 6. September 2011 konnte die Wechselkursstabilität bis Anfang 2015 gewährleisten. Die Aufhebung des Mindestwechselkurses am 15. Januar 2015 brachte, trotz Einführung des bis heute negativen Leitzinses in Höhe von minus 0,75 Pro- 
zent, in dem Jahr eine zweite Aufwertung um real effektiv rund 7,5 Prozent.

Die starke Aufwertung des Schweizer Frankens hatte jedoch nicht nur negative Auswirkungen auf die Wettbewerbsfähigkeit der exportorientierten Schweizer Wirtschaft, sondern verbesserte die Terms of trade so weit, dass sich derjenige Teil der Schweizer Wirtschaft, der nicht in direkter Konkurrenz zum Ausland steht, relativ stark entwickeln konnte. Zudem half die kräftige Erholung im Euroraum in den Jahren 2015 bis 2018 der Schweizer Wirtschaft, durch die Aufwertungsphase nach Aufhebung des Mindestwechselkurses zu kommen. Die 2019 gebremste globale Konjunktur und das schwache Wachstum der deutschen Industrie hemmten indes die Schweizer Wirtschaftsentwicklung. Bereinigt um die außerordentlichen Einnahmen aus großen internationalen Sportveranstaltungen, ergab sich eine Gesamtwachstumsrate von 1,4 Prozent. $^{3}$ Die durchschnittliche Wachstumsrate während des Zeitraums 2010-2018 und die von der KOF geschätzte Potenzialwachstumsrate für 2019 beliefen sich auf 1,9 beziehungsweise 1,75 Prozent.

Im Winter 2019/20 wurde eine Reduktion der internationalen Risiken im Zusammenhang mit dem Brexit und den Handelskonflikten, insbesondere zwischen den Vereinigten Staaten und China, erwartet. Damit schien eine wirtschaftliche Talsohle durchschritten und eine leichte Erholung im Jahr 2020 möglich zu sein. Wiederum bereinigt um die außerordentlichen Einnahmen aus großen internationalen Sportveranstaltungen, prognostizierte die KOF im Dezember 2019 daher für 2020 und 2021 Wachstumsraten von 1,4 Prozent und 1,8 Prozent (Abberger et al. 2019).

Bereits vor der Covid-19-Pandemie waren regionale Unterschiede in der wirtschaftlichen Situation der Schweizer Unternehmen sichtbar. Während die Geschäftslage in der Gesamtwirtschaft sowie in der konjunkturabhängigen Industrie zu Beginn des Jahres 2020 als überdurchschnittlich gut eingeschätzt wurde, meldeten die Unternehmen im Tessin und in der Genfersee-Region bereits vor dem Corona-Ausbruch ein Geschäftsklima unterhalb des Schweizer Durchschnitts (KOF Konjunkturumfrage 2020).

Im Verhältnis zu anderen OECD-Staaten war der fiskalische Spielraum der Schweiz am Anfang der Krise groß. Seit der Einführung der Schuldenbremse im Jahr 2001

3 Zur konjunkturellen Betrachtung der Schweizer Wirtschaft sind die unbereinigten BIP-Wachstumsraten nicht informativ. Alle zwei Jahre wiederkehrende Sportereignisse wie internationale Fußballmeisterschaften und die Olympischen Spiele - Fifa, UEFA und Olympisches Komitee haben ihren Sitz in der Schweiz - überlagern die für die Konjunktur relevanten Bewegungen. Die unbereinigte BIP-Wachstumsrate für das Jahr 2019 war 0,9 Prozent. durch eine Volksabstimmung mit einem Ja-Stimmenanteil von 85 Prozent, hat sich die Bruttoschuldenquote des Bundeshaushaltes vom Höchststand von 130,3 Milliarden (24 Prozent des BIP 2005) auf 96,9 Milliarden (14 Prozent des BIP 2019) reduziert. Zudem konnte sich die Eidgenossenschaft während der Corona-Krise wie auch in den vergangenen Jahren zu Negativzinsen verschulden. Die Gesamtverschuldung der öffentlichen Hand (einschließlich derjenigen der Kantone und Gemeinden) war 2019 zum ersten Mal seit Anfang der neunziger Jahre wieder unter 40 Prozent des BIP gefallen. Die seit der Finanzkrise nahezu kontinuierlichen Haushaltsüberschüsse im gesamten öffentlichen Sektor haben dies ermöglicht.

\section{3 Über welche Kanäle wirkt die Pandemie während eines Lockdowns auf die Konjunktur? ${ }^{4}$}

Die Covid-19-Pandemie wirkt sich über mehrere Kanäle auf die (Schweizer) Wirtschaft aus. In Bezug auf die volkswirtschaftlichen Auswirkungen muss zwischen nachfrageseitigen und angebotsseitigen Effekten unterschieden werden. Negative Nachfrageeffekte wie die Konsumzurückhaltung führen tendenziell dazu, dass die Preise und die Wertschöpfung der Unternehmen sinken. Negative angebotsseitige Effekte wie Lieferengpässe führen dagegen eher zu steigenden Preisen, weil die Unternehmen auf tendenziell teurere Vorleistungsgüter ausweichen müssen. Die Mehrkosten wälzen sie zum Teil auf die Verkaufspreise ab. Aus diesem Grund haben Nachfrage- und Angebotseffekte unterschiedliche Implikationen für die erwartete Erholung, die Inflationserwartungen und für die Art einer möglichen staatlichen Reaktion. Zudem stehen gesundheitspolitische Maßnahmen zur Eindämmung der Pandemie und wirtschaftspolitische Konjunkturmaßnahmen meist diametral zueinander. Der Einfluss der Erwartungen der Unternehmen und der Konsumenten über den nationalen und internationalen Verlauf der Epidemie auf ihr Investitions- und Konsumverhalten sind nicht zu unterschätzen.

\subsection{Nachfrageseitige Effekte}

Aufgrund von Sperrmaßnahmen, aus Unsicherheit über die persönliche finanzielle Situation und wegen der Sorge vor einer Ansteckung mit dem Virus, reduziert oder verschiebt

4 Dieser Abschnitt beruht auf Abberger et al. 2020, S. 7. 
die Bevölkerung ihren Konsum. Diese Konsumzurückhaltung trifft insbesondere konsumnahe Dienstleistungen und senkt das BIP zumindest vorübergehend. Wie stark dieser Wirkungskanal zum Tragen kommt, hängt von der Dauer und Entwicklung der Pandemie, der Substituierbarkeit, Verschiebbarkeit, dem Importanteil und dem Lagereffekt ab. Allerdings könnten Sorgen über die künftige wirtschaftliche Entwicklung und die eigene Einkommensentwicklung zu einer dauerhaft höheren Sparneigung führen.

Je geringer der Importanteil beziehungsweise je höher der inländische Wertschöpfungsanteil einer Güterkategorie ist, desto stärker wirkt sich die Konsumzurückhaltung in dieser Kategorie auf das inländische BIP aus. So dürfte die Konsumzurückhaltung beispielsweise besonders starke Effekte auf konsumnahe Dienstleister wie Friseure und Hausärzte haben. Branchen wie der Tourismus und der Einzelhandel werden den inländischen Wertschöpfungsanteil wohl aufgrund von reduzierten Dienstleistungsexporten temporär erhöhen können.

Kurzfristige Nachfrageveränderungen können die Firmen durch eine Anpassung der Lagerhaltung abfedern (Lagereffekt). Solche Effekte verteilen die Wirkung der Konsumzurückhaltung auf das BIP auf mehrere Quartale. Hier gibt es aber große Branchenunterschiede: Eventveranstalter und Restaurants beispielsweise können bei ausbleibender Nachfrage nicht auf Lager produzieren. Die erhöhte Lagerhaltung kann erhebliche Zusatzkosten für die Unternehmen bedeuten. Diese lohnen sich vor allem dann, wenn es nach der Lockerung der Maßnahmen starke Nachholeffekte gibt.

Auch wenn die Nachfrageeffekte im Aggregat eindeutig negativ sein werden, dürften infolge der Pandemie die Ausgaben in manchen Bereichen, zum Beispiel in Teilen des Gesundheitswesens und für Online-Dienstleistungen, deutlich steigen.

\subsection{Angebotsseitige Effekte}

Die Pandemie beeinträchtigt die Produktionsleistung der Unternehmen durch Produktionsstörungen und -verbote. Zunächst kann es wegen tatsächlicher Krankheitsfälle oder wegen prophylaktischer Maßnahmen dazu kommen, dass ein Teil der Arbeitnehmer nicht oder nur mit Einschränkungen arbeiten kann. Produktionsstörungen ergeben sich auch wegen Quarantänen von Mitarbeitern, der zwangsweisen Schließung von Geschäften und Unternehmensstandorten sowie der Sperrung von Grenzen und Schulen. Letztere zwingen Eltern, die etwa ein Sechstel der Beschäftigten in der Schweiz ausmachen, sowie Grenzgänger, rund 6 Prozent der Beschäftigten in der Schweiz, weniger $\mathrm{zu}$ arbeiten. Solche Maßnahmen mindern die Wertschöpfung zum einen direkt, weil die Unternehmen nicht in gleicher Weise produktiv sind, wenn die Arbeitnehmer dem Arbeitsplatz fernbleiben. Zum anderen wirken sie indirekt, weil Wertschöpfungsketten unterbrochen sind. Dadurch kann es zu Lieferschwierigkeiten mit Vorleistungsgütern kommen. So mussten beispielsweise Baustellen im Tessin aufgrund von Lieferengpässen geschlossen werden. Die Substitution von Vorprodukten oder ein Lieferantenwechsel ist für die meisten Unternehmen und Produktionsprozesse kurzfristig nur sehr schwer realisierbar. Die Wertschöpfungseffekte dieser Maßnahmen hängen einerseits davon ab, ob die Mitarbeitenden von $\mathrm{zu}$ Hause aus arbeiten können, andererseits aber auch davon, wie arbeitsintensiv die Produktion der betroffenen Betriebe generell ist. Insgesamt sind die Branchen den Produktionsausfällen unterschiedlich stark ausgesetzt.

Die Nachfragerückgänge und Produktionsstörungen führen zu Einnahmeausfällen der Unternehmen, wodurch es zu Liquiditätsengpässen kommen kann. Bei längerer Dauer der Krise kann dies eine Insolvenzwelle nach sich ziehen. Im schlimmsten Fall entsteht hieraus eine Finanzkrise - mit negativen Rückwirkungen auf die Realwirtschaft. Es ist daher allein schon wegen der Gefahr systemischer Risiken unerlässlich, dass sich die öffentliche Hand an den Fixkosten von Arbeit und Kapital beteiligt, die bei den betroffenen Unternehmen anfallen.

\subsection{Erwartungseffekte}

Die Erwartungsbildung der Unternehmen ist ein entscheidendes Element zur Beantwortung der Frage, ob die Pandemie die Wirtschaftsaktivität dauerhaft oder nur temporär beeinträchtigt und wie schnell sich die Wirtschaft wieder erholt. Zum einen dämpfen die Krisenberichte, Nachfragerückgänge und Produktionsstörungen die kurzbis mittelfristigen Geschäftserwartungen der Unternehmen in den meisten Branchen. Diese reagieren mit einer Reduktion oder Nicht-Ausweitung ihrer Produktionskapazitäten darauf. Zum anderen erhöht die Pandemie die Unsicherheit der Unternehmen über die künftige Geschäftsentwicklung. Als Konsequenz schieben die Unternehmen Personaleinstellungen und Investitionen auf. Beide Effekte drosseln die Investitions- und Beschäftigungsdynamik, was sich wiederum negativ auf die Investitionsentscheidungen anderer Unternehmen, den Arbeitsmarkt und die Kaufkraft auswirkt, wodurch sich die Krise verstärkt.

$\mathrm{Zu}$ Beginn der durch einen exogenen Schock ausgelösten Krise und insbesondere während der starken temporären Einschränkung des Wirtschaftslebens hat die Struktu- 
rerhaltung höchste Priorität. Massenentlassungen und eine große Anzahl von Insolvenzen aufgrund von Liquiditätsengpässen sind zu vermeiden. Es gilt der Gesellschaft und der Wirtschaft zu ermöglichen, temporär auf niedrigere Produktionsniveaus umzustellen. Soweit dies auch nur ansatzmäßig möglich ist, sollte die Regierung glaubwürdig über die Aussichten kommunizieren, damit die Wirtschaftsakteure Erwartungen bilden und sich entsprechend anpassen können.

Auch in der Lockerungsphase sind vorwärtsorientierende Aussagen (Forward guidance) wichtig, um den Unternehmen und deren Mitarbeitern eine gewisse Planungssicherheit zu geben oder zumindest die Produktionsbereitschaft aufrechtzuerhalten. Ökonomen betonen seit mehreren Jahren, dass Unsicherheit Gift für Unternehmen wie Haushalte ist. Die Regierungen können hier von den Zentralbanken lernen, welche die externe Kommunikation inzwischen als wichtiges Instrument zur Zielerreichung nutzen. Zwar kann es vollständige Sicherheit nicht geben. Doch es ist wichtig, mögliche Auswege zu kommunizieren, ohne dabei die Glaubwürdigkeit der Regierung und ihrer Ankündigungen zu gefährden. Das schafft nicht nur Vertrauen, sondern lindert auch die Unsicherheit. Während des Lockdowns können unklare Aussichten eine Resignation der Bürger befördern, und während der Phase, in der eine Normalisierung gewünscht wird, bremst fehlende Zuversicht die wirtschaftliche Aktivität.

\section{Wirtschaftspolitische Maßnahmen}

In wirtschaftspolitischen Szenarien setzen sich die gesamtwirtschaftlichen Kosten für die Schweiz aus zwei Hauptkomponenten zusammen: Einerseits aus den unbeeinflussbaren Kosten, die durch das internationale Umfeld und die mit Covid-19 zusammenhängenden Krankheitsausfälle verursacht werden, und andererseits aus den durch inländische Maßnahmen bedingten Wertschöpfungsverlusten. Per Definition können wirtschaftspolitische Maßnahmen im Inland die international verursachten Kosten nur abfedern, das heißt, innerhalb der Gesellschaft verteilen.

$\mathrm{Zu}$ Beginn der Covid-19-Krise und insbesondere während des Lockdowns ging es um Strukturerhaltung, was vor allem mit Kurzarbeit und zinsfreien und (zu großen Teilen) staatlich garantierten Krediten zur Liquiditätserhaltung erreicht werden sollte. Während die Maßnahmen an sich bereits von Beginn an im Großen und Ganzen geeignet waren, wurden die von der öffentlichen Hand zur
Verfügung gestellten Beträge nur zögerlich und schrittweise erhöht. Eine bessere Forward guidance hätte hier wohl unnötige Sorgen verhindert, ohne Mehrkosten zu verursachen. Mögliche Mitnahmeeffekte (Moral hazard) bei den breit angelegten Maßnahmen mit vereinfachten Verfahren wurden durch die Ankündigung einer späteren ausführlichen Prüfung der während der Krise nur oberflächlich prüfbaren Anträge bekämpft (vgl. NZZ 2020). Dass kleinere und mittlere Unternehmen (KMU) keinerlei Unterstützung für ihre Kapitalkosten erhalten haben, sehen wir ebenfalls kritisch.

\subsection{Arbeitsmarkt}

Die erste und bis heute wichtigste Säule des Maßnahmenpakets ist die Kurzarbeit. Oberstes Ziel der Kurzarbeit ist es, Entlassungen und damit Arbeitslosigkeit zu verhindern. Kurzarbeit kann von Unternehmen beantragt werden, die mit einem vorübergehenden Rückgang der Nachfrage nach ihren Gütern und Dienstleistungen konfrontiert sind. Durch Kurzarbeit in Teil- oder Vollzeit wird diesen Betrieben ermöglicht, die Arbeitszeit ihrer Mitarbeitenden vorübergehend zu reduzieren. Die betroffenen Mitarbeitenden werden in der Schweiz von der Arbeitslosenversicherung zu 80 Prozent für den Lohnverlust entschädigt. In einer 2018 veröffentlichten KOF-Studie zeigen Kopp und Siegenthaler (2018), dass die Kurzarbeit zwischen 2009 und 2015 ein effektives Instrument war, um Entlassungen langfristig zu verhindern. Die erzielten Einsparungen an Arbeitslosengeldern dürften dabei groß genug gewesen sein, um die zusätzlichen Ausgaben für Kurzarbeitsentschädigungen zu decken.

Am 13. März 2020 wurden zunächst 8 Milliarden Franken für die Kurzarbeitsentschädigung bereitgestellt und bereits am 20. März substanziell aufgestockt. Der Bundesrat kündigte am 20. März zudem die Ausweitung und Vereinfachung der Kurzarbeit und eine Entschädigung der Erwerbsausfälle von Selbständigen an (Bundesrat 2020c). Abbildung 2 zeigt die großen kantonalen Unterschiede im Anteil der Beschäftigten, für die Kurzarbeit angemeldet wurde. Ins Auge fallen insbesondere der Kanton Tessin (TI) im Süden des Landes, der eine besonders hohe Anzahl Infizierte pro Kopf der Bevölkerung verzeichnete und zudem stark vom (innerschweizerischen) Tourismus abhängt. Auf dringliche Empfehlungen des Bundesrats und des Kanton Tessins sollten die Bürger zum Saisonstart um Ostern auf Reisen in das Tessin aber verzichten. Die Kantone Jura (JU) und Neuchâtel (NE) sind stark von der Uhrenindustrie abhängig, die besonders hart vom globalen Nachfragerückgang betroffen sein dürfte. 


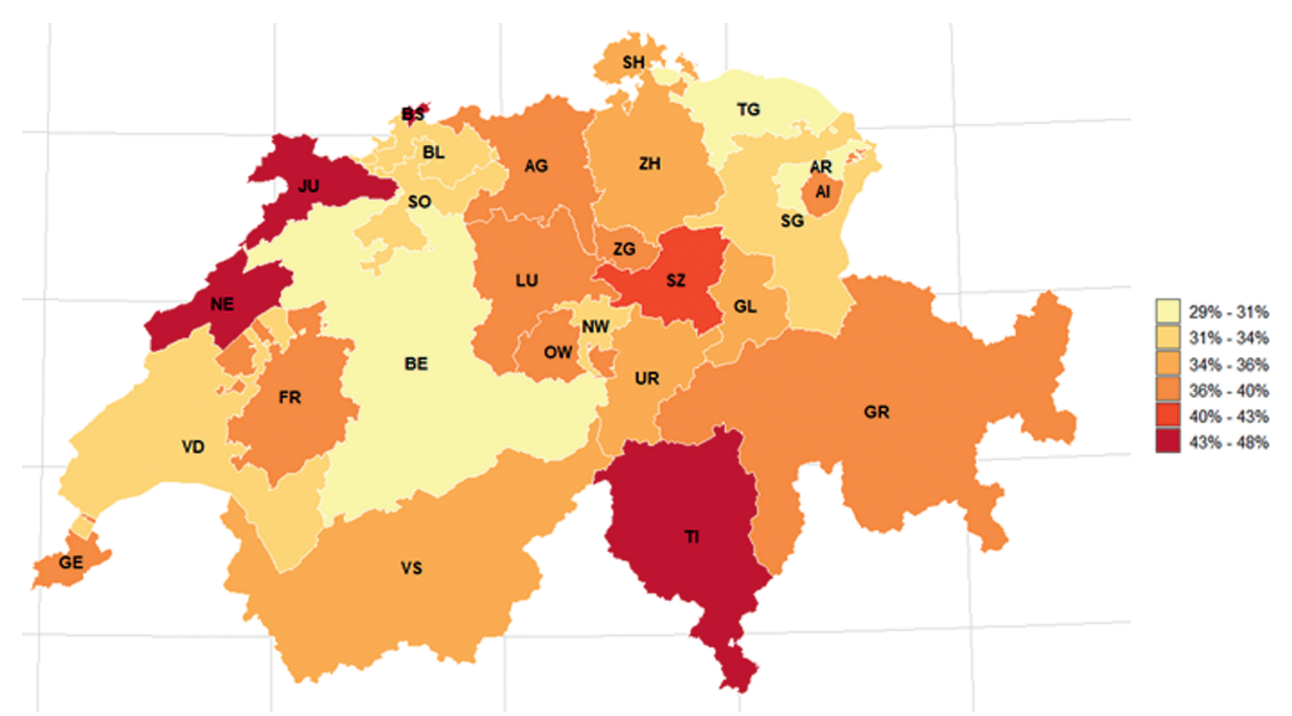

Abbildung 2: Anteil der für Kurzarbeit vorangemeldeten Arbeitnehmer nach Kanton Ende April 2020 Quelle: Darstellung der KOF basierend auf Zahlen des Staatssekretariats für Wirtschaft (SECO)

\subsection{Liquidität der Unternehmen}

Die zweite Säule der Strukturerhaltung erlaubt es Unternehmen, staatlich garantierte Kredite aufzunehmen. Die Liquiditätshilfen für KMU im Umfang von bis zu 20 Milliarden Franken (ab 3. April 40 Milliarden Franken) wurden am 20. März angekündigt und konnten ab dem 26. März bei der jeweiligen Hausbank beantragt werden. ${ }^{5}$ Die zinsfreien Darlehen sind staatlich garantiert; bis zu einer halben Million Franken zu 100 Prozent und für darüberhinausgehende Beträge zu 85 Prozent (Bundesrat 2020b). Die Laufzeit beträgt fünf Jahre, kann jedoch auf maximal sieben Jahre verlängert werden. Die Kredite dürfen maximal 20 Millionen Franken oder 10 Prozent des Vorjahresumsatzes betragen. Letzteres ist kritisch zu sehen, da die Bedeutung des Umsatzes zwischen Wirtschaftszweigen und Geschäftsmodellen bekanntlich stark variiert. Die Liquiditätskredite sind auf eine große Nachfrage gestoßen: Bis zum 29. April 2020 wurden von 123 Banken mehr als 117.000 Kreditvereinbarungen abgeschlossen, die sich bereits auf rund 16,1 Milliarden Franken summieren (Abbildung 3). Der durchschnittliche Bürgschaftsbetrag beträgt 131.000 Franken.

Die Unternehmen erhielten zudem einen zinsfreien Zahlungsaufschub für die Sozialversicherungsbeiträge. Auch wurden die Zahlungsfristen für Steuern und Abgaben an staatliche Dienstleister verlängert, ohne dass Zinsen auf die verspäteten Zahlungen fällig werden. Diese

5 Bereits ab dem 13. März standen via Bürgschaftsorganisationen 580 Millionen Franken zur Verfügung (Bundesrat 2020a).
Maßnahmen sind als kurzfristige Kredite des Staates zu sehen und gehören somit zur zweiten Säule. Zudem wurde im Betreibungswesen (Gerichtsvollzieher) ein Rechtsstillstand vom 19. März bis einschließlich 4. April 2020 festgelegt. Für den Sport- und Kultursektor sowie die Tourismusindustrie wurden Hilfen in Höhe von mehreren Millionen Franken versprochen.

Für Unternehmen mit mehr als 500 Millionen Franken Umsatz sind bisher keine staatlichen Liquiditätsmaßnahmen vorgesehen. Eine Ausnahme ist die bereits am 8. April angekündigte Prüfung einer möglichen Unterstützung der Luftfahrtbranche. Am 29. April beantragte der Bundesrat beim Parlament 1,275 Milliarden Franken, davon 1,2 Milliarden Franken für die Airline Swiss, einer Tochtergesellschaft der Lufthansa. Die Kredite wären wiederum von den Banken zu vergeben, welche 15 Prozent des Kreditrisikos zu tragen hätten (Bundesrat 2020d). Die staatlich garantierten Darlehen können, anders als im Fall der KMU, bis zu einem Viertel des Umsatzes der Swiss ausmachen. Der Bundesrat hält fest, dass diese Mittel nur den schweizerischen Infrastrukturen dienen dürfen und in der Zukunft erwirtschaftete Mittel prioritär zur Rückzahlung der Liquiditätshilfen zu verwenden sind. ${ }^{6}$ Die Kredite der Eidgenos-

6 Diese Kredite hängen von der Unterstützung Deutschlands für die Lufthansa ab. Für die flugnahen Betriebe, die in ausländischer Hand sind, wurde keine Maßnahme beschlossen, jedoch ein Kredit in Höhe von 600 Millionen Franken beantragt. Bei Easyjet Switzerland sind aufgrund der Liquidität des Mutterkonzerns die Bedingungen für ein subsidiäres Engagement der Eidgenossenschaft gegenwärtig nicht gegeben. 


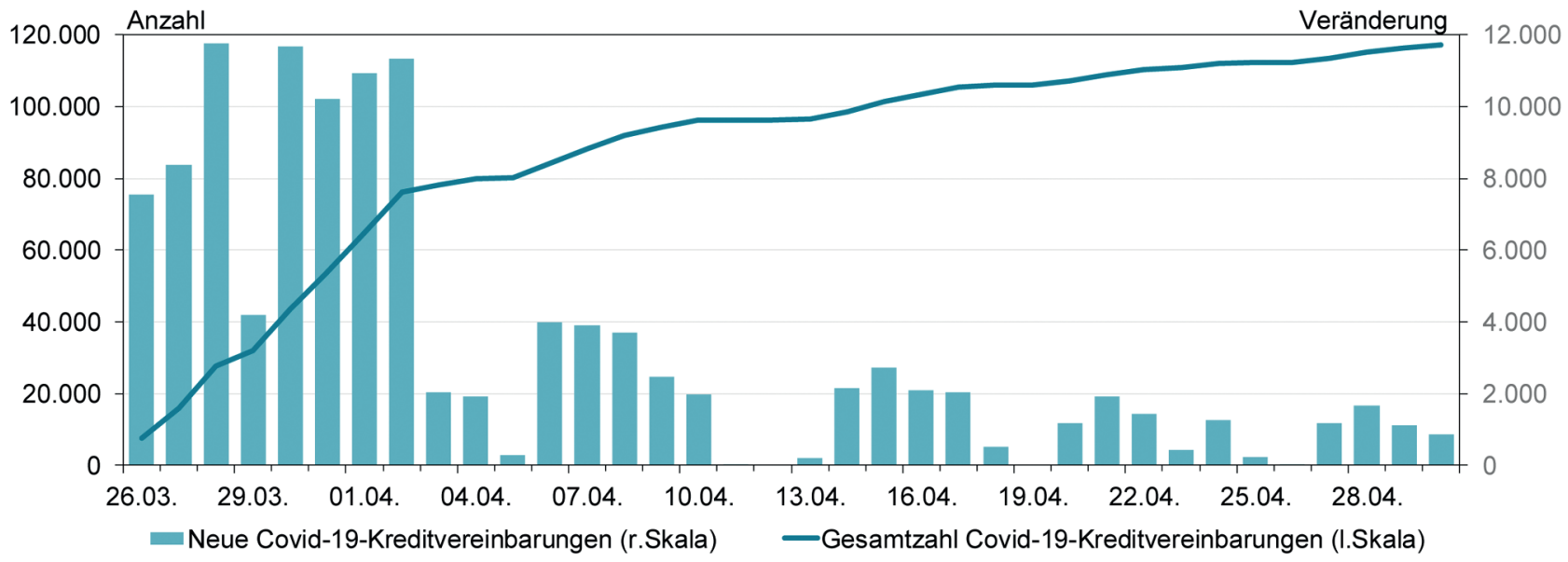

Abbildung 3: Covid-19-Kreditvereinbarungen kumuliert seit Implementierung am 26. März

Quelle: Eigene Darstellung, basierend auf online verfügbaren Daten unter https://covid19.easygov.swiss/fuer-medien/

senschaft hängen von der Unterstützung Deutschlands für die Lufthansa ab.

Zur Sicherung der Liquidität des Finanzsystems schuf die Schweizerische Nationalbank (SNB) am 25. März eine temporäre SNB-Covid-19-Refinanzierungsfazilität (CRF) für Banken in der Schweiz und in Liechtenstein (SNB 2020). Dadurch kann Liquidität als gedecktes Darlehen gegen Forderungen aus Krediten der Covid-19-Solidarbürgschaftsverordnung und gegen weitere von der SNB zugelassene Sicherheiten bezogen werden. Das Darlehen ohne fixe Laufzeit wird mit dem SNB-Leitzins verzinst.

\subsection{Evaluierung}

Die Regierung hat im April Anpassungen an beiden Säulen der Strukturerhaltung vorgenommen, wodurch sich das geschätzte Maximalvolumen der Maßnahmen auf 95 Milliarden Franken erhöht hat (Blick 2020), was eine Verdoppelung der Schulden der Eigenossenschaft bedeuten würde. Dennoch reichen diese Maßnahmen nach Einschätzung vieler Ökonomen nicht aus. Denn die Unternehmen mit geringen oder keinen Einnahmen haben weiterhin Fixkosten wie Miete, Pacht oder Kreditzinsen zu tragen. Diese Kapitalkosten werden mit den beiden Säulen der Strukturerhaltung nicht aufgefangen und sollten den betroffenen Unternehmen über bestehende Kanäle teilweise erstattet werden. Wenn die Unternehmen diese Kapitalkosten allein tragen müssen, besteht die Gefahr, dass viele von ihnen insbesondere KMU - den Betrieb einstellen oder aufgrund der hohen Schuldenlast nach der Krise kaum investieren. Dies bremst die wirtschaftliche Dynamik auf längere Sicht.

Am 31. März haben deshalb Hans Gersbach und JanEgbert Sturm (2020b) einen dritten Stützpfeiler gefordert: ein „Kurzarbeits-Regime für das Kapital“. Basierend auf ihren Überlegungen für einen großen Rettungsschirm schlagen die Ökonomen der ETH Zürich vor, dass der Staat Unternehmen die Kapitalkosten bei einem Produktionsausfall teilweise erstattet. Ähnliche Vorschläge kamen von Ökonomen der Universität Zürich (Alós-Ferrer et al. 2020) und aus der Westschweiz (Bonardi et al. 2020). Als Grundlage könnten Miet- und Pachtverträge oder die vor dem Ausbruch des Coronavirus gezahlten Kreditzinsen dienen. Auch eine eher pauschale Lösung über eine teilweise Rückerstattung von Mehrwertsteuern für andauernde Produktionseinbrüche ist möglich. Für die praktische Umsetzung bieten sich die Kanäle an, die bereits für die Kurzarbeitsentschädigung und die Liquiditätshilfen genutzt werden. Aufgrund der niedrigen Schuldenquote der Eidgenossenschaft und der Negativzinsen auf Staatsanleihen hat die Schweiz, im Gegensatz zu anderen Industriestaaten, den fiskalpolitischen Spielraum für solche Maßnahmen.

Zudem hätte man den Wirtschaftsakteuren früh eine möglichst umfassende Perspektive geben können, auch ohne spezifische Maßnahmen zu nennen und natürlich ohne eine Vollkaskoversicherung für die Wirtschaft $\mathrm{zu}$ suggerieren. In diesem Kontext ist der Vorschlag der Ökonomen Hans Gersbach und Jan-Egbert Sturm (2020a) zu sehen, die bereits am 18. März die Einrichtung eines Schweizfonds im Volumen von 100 Milliarden Franken anregten. Die Ankündigung einer solchen Maßnahme hätte wohl zusätzliche Zuversicht verbreitet. Abbildung 4 zeigt, dass sich die Angst um die wirtschaftliche Existenz bei vergleichbaren Unternehmen stark veränderte, sobald Informationen zur Lockerungsstrategie bekannt wurden.

Trotz der umfassenden Maßnahmen im In- und Ausland überrascht es kaum, dass der KOF Geschäftslageindikator im April außerordentlich stark gesunken ist (Abbildung 5). Vor dem Hintergrund der Covid-19-Pandemie 


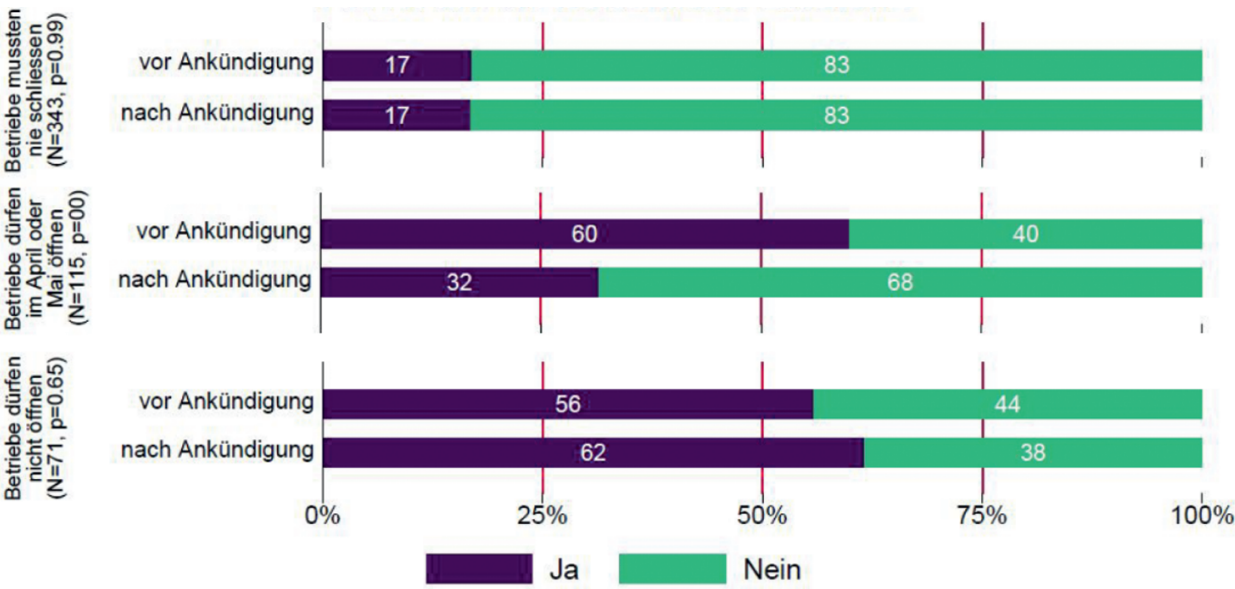

Abbildung 4: Angst um die wirtschaftliche Existenz vor und nach der Lockerungsankündigung Anmerkung: Gruppierung danach, ob in der bundesrätlichen Ankündigung vom 16. April eine Öffnung des Betriebes in Aussicht gestellt wurde (Bundesrat 2020b). Untergruppierungen danach, ob die Umfrage vor der bundesrätlichen Ankündigung ausgefüllt wurde oder nachher. Öffnung im April oder Mai: Alle Läden, nicht-dringliche medizinische Behandlungen, Baumärkte, Blumenläden, Gartenzentren, Gärtnereien und Dienstleistungen der Körperpflege. Keine Information über Zeitpunkt der Öffnung: Alle Betriebe aus Tourismus, Hotellerie und Gastronomie sowie Sport und Kultur, die schließen mussten oder deren Tätigkeit stark eingeschränkt wurde.

Quelle: Brülhart et al. 2020

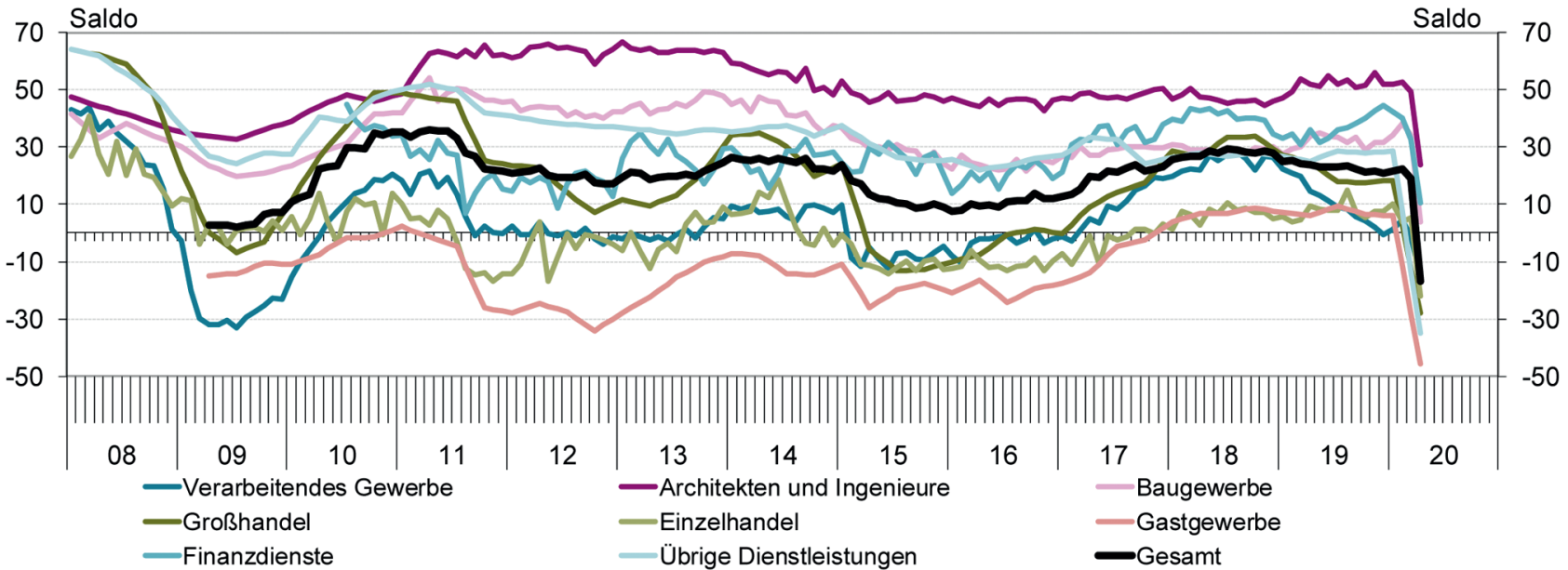

Abbildung 5: Geschäftslage nach Wirtschaftszweigen

Anmerkung: Der Saldowert ist der Prozentanteil der „gut“-Antworten minus dem Prozentanteil der „schlecht“-Antworten auf die Frage nach der Beurteilung der aktuellen Geschäftslage.

Quelle: KOF Konjunkturumfrage

bewerten die Unternehmen ihre Geschäftslage noch negativer als während der Finanzkrise 2009. Die Krise hinterlässt in allen Wirtschaftszweigen tiefe Spuren.

Die KOF Konjunkturumfrage vom April 2020 gibt zudem erste Hinweise über die wirtschaftlichen Herausforderungen während der Lockdown-Phase. So scheint der Nachfrageeinbruch schwerer zu wiegen als die Behinderung im eigenen Betriebsablauf. Auf eine Zusatzfrage nach der expliziten Nachfragewirkung der Pandemie geben 55 Prozent der Unternehmen an, dass die Nachfrage nach ihren Leistungen deutlich reduziert ist. Sowohl im Teilbereich Verkehr, Information, Kommunikation als auch bei den wirtschaftlichen und den persönlichen Dienstleistungen sind die Nachfrageerwartungen negativer als während der Finanzkrise. Gefragt nach den Beschränkungen durch die Einsatzfähigkeit des eigenen Personals, geben 36 Prozent der Firmen an, dass sie dadurch stark beeinträchtigt sind. Die Banken erwarten einerseits, angestoßen durch die Hilfsmaßnahmen der öffentlichen Hand, ein deutlich zunehmendes Kreditgeschäft mit Firmenkunden, jedoch bei erheblich verschlechterter Bonität. Andererseits rechnen sie mit weniger starken Zuwächsen im Kreditgeschäft mit Privatkunden. 


\section{Schlussfolgerungen und abschließende Bemerkungen}

$\mathrm{Zu}$ Beginn der Covid-19-Krise war die allgemeine Auffassung oder zumindest Hoffnung, dass die Schweiz nach einer kurzen Phase des Stillstands und der internationalen Abschottung eine V-förmige Erholung erleben würde. Man setzte darauf, dass man leicht und schnell zu einer wirtschaftlichen Normalität zurückkehren könne, wie sie vor der Krise herrschte. Diese Sichtweise ändert sich mehr und mehr. Die Diskussion hat sich zunehmend in Richtung einer U-, einer Wurzelzeichen- oder sogar einer L-förmigen Entwicklung der Gesamtwirtschaft bewegt. Inzwischen werden immer mehr strukturelle Veränderungen und damit zusätzliche Verteilungsfragen erwartet.

Die Frage, wie die Lasten innerhalb der Gesellschaft verteilt und insbesondere die Verlierer kompensiert werden können, wird damit immer wichtiger. Im Großen und Ganzen ist die öffentliche Hand als Versicherer aufgetreten und hat zunächst einen beträchtlichen Teil der (Personal-) Kosten auf sich genommen. Aber wie wird die (verbleibende) Last zwischen den Firmeninhabern und ihren Gläubigern aufgeteilt? Wie fängt man die Verlierer auf der Haushaltsseite auf? In dem Maße, in dem der Staat die Last auf sich genommen hat und weiter nehmen wird, ist klar, dass die höheren Schulden, die sich daraus ergeben, früher oder später zurückgezahlt werden müssen. Inwieweit dies die gegenwärtige oder künftige Generation tun muss, ist ebenso offen wie die Verteilung innerhalb einer Generation.

Es ist leicht zu begründen, dass Regierungen die Unternehmen während der Lockdown-Phase unterstützen sollten. Ein gesellschaftlich gewollter Winterschlaf oder zumindest Rückgang der Produktion sollte auch gesellschaftlich getragen werden. Die Wirtschaftsstrukturen sollten während einer solchen Phase erhalten bleiben, um eine schnelle Erholung und Normalisierung zu ermöglichen. Mit dem Ende behördlich verordneter Sperrmaßnahmen und Einschränkungen wird es jedoch zunehmend schwieriger zu begründen, wo staatliche Unterstützung benötigt wird und wo strukturelle Veränderungen zugelassen werden sollten. Dies hängt stark von den mittel- bis längerfristigen Aussichten für die Entwicklung einzelner Wirtschaftszweige ab. Gibt es bleibende Verhaltensänderungen beispielsweise im Tourismus und im Zusammenhang mit anderen Freizeitaktivitäten?

Der krisenbedingte Verschuldungsanstieg von öffentlicher Hand und Privatunternehmen wird die wirtschaftliche Erholung jedes Landes belasten. Weiterhin unklar ist hingegen, ob primär die angebots- oder nachfrageseitigen
Effekte die Erholung bremsen werden. Mit einer relativ allgemein gehaltenen Forward guidance über mögliche staatliche Unterstützung können Regierungen erstens die wirtschaftliche Erholung gleichsam gratis stimulieren und zweitens, bei Bedarf, die geeigneten wirtschaftspolitischen Maßnahmen ergreifen, wenn sich die Probleme klarer darstellen. Das Ausmaß der staatlichen Unterstützung kann nicht nur aus einer nationalen Perspektive betrachtet werden, sondern bedarf sicherlich auch einer Form der internationalen Koordinierung. Der internationale Austausch sollte auch die Forward guidance umfassen. Ansonsten besteht das Risiko, dass die staatliche Unterstützung bis hin zu keynesianischen Stimulierungsmaßnahmen sowohl $\mathrm{zu}$ einem inländischen Lobbying-Boom zwischen den Branchen als auch zu einem internationalen Konkurrenzkampf zwischen den Nationen führt. Wir könnten uns so in einem Gefangenendilemma wiederfinden, in dem am Ende unterlassen wird, was für alle besser gewesen wäre.

Wie erwähnt, steht die öffentliche Hand in der Schweiz trotz der Krise noch immer verhältnismäßig gut da. Die Staatsverschuldung war gering, die Haushaltsdisziplin groß. Im Vergleich $\mathrm{zu}$ anderen Ländern wird in der Schweiz somit weniger über eine Monetarisierung der Kosten diskutiert. Aufgrund der internationalen Verflechtungen und der Rolle des Wechselkurses für das Wohlergehen der Schweizer Wirtschaft interveniert die Schweizerische Nationalbank auf den Wechselkursmärkten und häuft weitere internationale Reserven an. Die Bilanz der SNB steigt damit weiter über die bereits Ende 2019 deutlich überschrittenen 110 Prozent des Schweizer BIP.Zudem versorgt sie das inländische Bankensystem mit ausreichend Liquidität, um die Stabilität des Schweizer Finanzsystems aufrecht zu erhalten. In einer Welt, in der viele und vor allem die größeren Zentralbanken in der quantitativen Lockerung immer weitergehen und in einigen Fällen damit beginnen, Staatsausgaben direkt zu finanzieren, stellt sich die Frage, ob oder wie lange die aktuelle geldpolitische Strategie einer relativ kleinen Zentralbank wie der SNB noch aufgehen kann.

\section{Literaturverzeichnis}

Abberger, K. et al. (2019), Konjunkturanalyse: Prognose 2020/2021, Schwächephase hält an, KOFAnalysen 4, S. 1-28.

Abberger, K. et al (2020), Konjunkturszenarien, Frühjahr 2020: Im Bann des Coronavirus Rezession in Europa und der Schweiz wahrscheinlich, KOFAnalysen 1, S. 1-62.

Alós-Ferrer, C. et al. (2020), Coronavirus - Testen und Einfrieren: Eine Überlebensstrategie für die Schweizer Volkswirtschaft, Position Paper, Universität Zürich, online verfügbar unter https://www. 
econ.uzh.ch/dam/jcr:01ef12ba-a183-4667-9f.660a33a67d182/20200329_Corona_Positionspapier.pdf.

Blick (2020), Die Corona-Rechnung von Ueli Maurer: Wir zahlen auch 2030 noch dafür, online verfügbar unter https://www.blick.ch/ news/politik/ausserordentliches-defizit-bis-zu-55-milliardenfranken-die-corona-rechnung-von-ueli-maurer-wir-zahlen-auch2030-noch-dafuer-id15865652.html.

Bonardi, J.-P., M. Brülhart, J.-P. Danthine, E. Jondeau und D. Rohner (2020), Wieso und wie der Staat die wirtschaftlichen Kosten des Lockdown übernehmen sollte: Lohnfortzahlungen und CoronaDarlehen, Batz.ch.

Brülhart, M., J. Kläui, R. Lalive, T. Lehmann und M. Siegenthaler (2020), Die Schweizer Selbständigerwerbenden in der Covid19Pandemie, 3. Mai 2020, online verfügbar unter https://ethz.ch/ content/dam/ethz/special-interest/dual/kof-dam/documents/ Medienmitteilungen/Sonstige/Hintergrundbericht_Selbstaendigenumfrage.pdf.

Bundesrat (2020a), Bundesrat verschärft Massnahmen gegen das Coronavirus zum Schutz der Gesundheit und unterstützt betroffene Branchen, Medienmitteilung vom 13. März 2020, online verfügbar unter https://www.admin.ch/gov/de/start/dokumentation/medienmitteilungen.msg-id-78437.html.

Bundesrat (2020b), Bundesrat lockert schrittweise Massnahmen zum Schutz vor dem neuen Coronavirus, Medienmitteilung vom 16. April 2020, online verfügbar unter https://www.admin.ch/ gov/de/start/dokumentation/medienmitteilungen.msg-id78818.html.

Bundesrat (2020c), Coronavirus: Massnahmenpaket zur Abfederung der wirtschaftlichen Folgen, Medienmitteilung vom 20. März 2020, online verfügbar unter https://www.admin.ch/gov/de/ start/dokumentation/medienmitteilungen.msg-id-78515.html.

Bundesrat (2020d), Coronavirus: Bundesrat konkretisiert Unterstützung für Luftfahrtunternehmen, Medienmitteilung vom 29. März 2020, online verfügbar unter https://www.admin.ch/gov/de/ start/dokumentation/medienmitteilungen.msg-id-78944.html.
Gersbach, H. und J.-E. Sturm (2020a), Ein Schweizfonds mit 100 Mia. Franken als zweiter Pfeiler, Ökonomenstimme, online verfügbar unter https://www.oekonomenstimme.org/artikel/2020/03/ ein-schweizfonds-mit-100-mia.-franken-als-zweiter-pfeiler/.

Gersbach und Sturm (2020b), „Kurzarbeits-Regime für Kapital“ als dritter Pfeiler, Ökonomenstimme, online verfügbar unter https://www.oekonomenstimme.org/artikel/2020/03/kurzarbeits-regime-fuer-kapital-als-dritter-pfeiler/.

KOF Konjunkturforschungsstelle (2020), Prognose Indikatoren, Stand 29. April 2020, online verfügbar unter https://kof.ethz.ch/prognosen-indikatoren/indikatoren.html.

Kopp, D. und M. Siegenthaler (2018), Does short-time work prevent unemployment? KOFStudies 106.

NZZ - Neue Zürcher Zeitung (2020), Corona-Kredite für KMU: Wie der Bund Betrüger abschreckt, online verfügbar unter https://www. nzz.ch/wirtschaft/covid-kredite-fuer-kmu-wie-leicht-kann-manden-bund-an-der-nase-herumfuehren-ld.1551774.

Rathke, A., S. Sarferaz, S. Streicher und J.-E. Sturm (2020a), SzenarioAnalysen zu den kurzfristigen wirtschaftlichen Auswirkungen der COVID-19-Pandemie, Medienmitteilung der KOF Konjunkturforschungsstelle, online verfügbar unter https://ethz.ch/ content/dam/ethz/special-interest/dual/kof-dam/documents/ Medienmitteilungen/Prognosen/2020/Corona_Krise.pdf.

Rathke, A, S. Sarferaz, S. Streicher und J.-E. Sturm (2020b), Update: Kosten des Lockdown und nächste Publikationen, Mitteilung der KOF Konjunkturforschungsstelle, online verfügbar unter https:// kof.ethz.ch/news-und-veranstaltungen/news/kof-news0/ 2020/04/update-kosten-des-lockdown-und-naechste-publikationen.html.

SNB - Schweizerische Nationalbank (2020), Nationalbank schafft SNB-COVID-19-Refinanzierungsfazilität und stellt Antrag auf Deaktivierung des antizyklischen Kapitalpuffers, Medienmitteilung vom 25. März 2020, online verfügbar unter https://www. snb.ch/de/mmr/reference/pre_20200325/source/ pre_20200325.de.pdf. 